\title{
DOCTRINA
}

\section{Reducción de los desechos plásticos en Chile: Elementos para profundizar nuestra regulación}

\author{
Plastic waste reduction in Chile: Elements to deepen our regulation
}

\section{Camilo Cornejo Martínez (iD)}

Facultad de Derecho, Universidad de Chile.

\begin{abstract}
RESUMEN Los antecedentes muestran que los desechos plásticos están profundizando la crisis climática y generando serias vulneraciones a derechos fundamentales, tales como la vida, salud y medio ambiente sano, todo ello con un fuerte impacto económico. La industria y los consumidores no han mostrado una reacción, cuestión que exige considerar una regulación adecuada que gestione el problema. La adopción de medidas requiere ponderar distintos bienes jurídicos, para lo que la teoría constitucional puede aportar elementos de análisis, como el sistema de ponderación de derechos o la eficacia en el uso de recursos públicos. Al mismo tiempo, principios ambientales tales como la proscripción de la regresión, la promoción de la progresividad y la prevención de daños ambientales moldean la respuesta. En cuanto a los residuos plásticos, se puede ver el mismo movimiento regulatorio y la promoción de medidas que prohíben ciertos plásticos a nivel internacional, comparado, regional y local. Esta reacción global evidencia como un punto de partida, la prohibición de los plásticos de un solo uso, para lo cual existe toda una experiencia que puede ser utilizada como referencia. Con todo, incluso sin una regulación sobre la materia y solo considerando el ordenamiento vigente, hay una necesidad jurídica de adoptar medidas contra este problema.
\end{abstract}

PALABRAS CLAVE Desechos plásticos, prohibición de plásticos de un solo uso, regulación, derechos fundamentales.

ABSTRACT Data shows that plastic waste is aggravating climate crisis and it is generating serious violations of fundamental rights such as life, health, and the right to a healthy environment, all with a strong economic impact. Industry and consumers have not shown any reaction, in an issue that requires considering an adequate regulation to manage the problem. The adoption of measures needs to take into account different legal assets. In this, constitutional theory can provide elements of analysis, such as the system of balancing rights or the effectiveness in the use of public resources. At the same time, the legal response should be shaped by environmental principles, such as non- 
regression, promoting progressivity and preventing environmental damages. Regarding plastic waste, we can see the same regulatory movement at an international level, comparative, regional and local systems. In the legal reaction, one start point to consider is the prohibition of single-use plastics. However, even in a scenario with a lack of regulation and only considering our actual legal system, we already find ourselves in the legal need to adopt measures against the problem of plastic waste.

KEYWORD Plastic waste, prohibition of single use plastic, regulation, fundamental rights.

\section{Introducción}

El plástico, con sus cortos sesenta o setenta años de vida, está presente prácticamente en todos los quehaceres, desde exploraciones planetarias hasta un simple envoltorio. Su base sintética, naturaleza dúctil, resistente, adaptable y barato para sus productores, lo llevó a ser presentado como el material de los mil usos (Téllez, 2012). Fue indicado como salvador de elefantes o protector de bosques (dadas sus características, era más barato que el marfil, lo que evitaría la matanza del noble animal o la tala indiscriminada de árboles).

Con el tiempo, se masificó y comenzó a ser asociado como un producto sin mayor valor y de rápido desecho (Oceana, 2020). Esta percepción repercutió en su nivel de producción, la que aumentó exponencialmente cada año, instalándolo como un producto cotidiano en el boom del consumismo y la cultura del desecho.

Hoy es apuntado como uno de los materiales que está generando un gran colapso en los océanos y su fauna, un ambiente central para la estabilidad de los ciclos planetarios. ${ }^{1}$ Su acumulación como desecho está generando un gran caos en toda clase de ambientes y para todos los integrantes de las cadenas alimenticias, incluidos los humanos que, por distintos puntos de contacto, estarían ingiriendo cada vez mayores cantidades del material, con efectos aún inciertos.

Las grandes dimensiones y profundidad del problema han forzado una reacción del sistema jurídico ante la producción incontrolada de plástico, en lo que se ha promovido la prohibición de plásticos de un solo uso, esto es, aquellos plásticos que una vez que llegan al consumidor final, son rápidamente desechados por este, entre los que se encuentran envases, botellas, envoltorios u otros. En el ordenamiento del país, la discusión puede tomar distintos ribetes. Por ello, desde una consideración del sistema internacional comparado y los principios constitucionales se analizará la

1. Las crudas imágenes de tortugas estranguladas por plástico, aves e incluso ballenas muertas por inanición al ingerirlo como alimento, verdaderas islas que deambulan en nuestros océanos, son solo alguna de las consecuencias que hoy son difundidas en distintos medios. 
respuesta del sistema chileno, rescatando la discusión legislativa y algunos elementos que podría considerar; pero más que nada, proponiendo que incluso sin la dictación de una nueva norma - que por lo demás parece necesaria- ya es posible que los distintos actores adopten medidas y se responsabilicen por el uso indiscriminado del material, cada vez que existen elementos jurídicos que orientan su actuación.

Para lo anterior, esta propuesta pretende rescatar el movimiento que gira en torno a la reducción de los desechos plásticos y en particular la prohibición de los denominados plásticos de un solo uso y las consideraciones normativas que orientan esta reacción. Para ello, 1) se sistematizarán algunos de los efectos de los desechos plásticos; 2) se estudiará la reacción internacional y comparada, para luego, 3) considerar la situación nacional, examinando tanto las normas existentes como los proyectos de ley en tramitación. Hecho esto, 4) se cerrará con algunas conclusiones sobre la medida.

\section{Aproximándonos al problema de los plásticos de un solo uso}

La presencia de los desechos plásticos en el ambiente produce una serie de efectos, cualquiera sea la escala desde la cual se haga una aproximación: a) afecta a personas, b) economías, c) sistemas de vida, d) a la naturaleza, e) generaciones futuras, y f) también está relacionado con el cambio climático.

Si se pone atención en las consecuencias sobre las personas, diversos estudios han detectado la presencia del material en el organismo. Solo para hacerse una idea, se ha reportado que los humanos en promedio estarían consumiendo una tarjeta de crédito a la semana, por plástico que proviene del aire, agua y comida que ingieren. Sus efectos en el organismo están recién comenzando a ser documentados:

[Pero está] comprobado que algunos de estos materiales afectan la función sexual, la fertilidad y un aumento en la incidencia de mutaciones y cáncer. Los microplásticos portados por el aire también tienen contaminantes del ambiente circundante. En los ambientes urbanos pueden llevar metales e hidrocarburos aromáticos policíclicos (HAP) - moléculas encontradas en el carbón y el alquitrán (Dalberg Global Development Advisors, y The University Of Newcastle, 2019: 11).

Pero no solo la salud de las personas, sino que también la economía se ve impactada. La pesca está sufriendo un gran estrés con la afectación de los recursos marinos, por su parte la industria del turismo es otra de las grandes amenazadas por los desechos plásticos: «Tailandia cerró Maya Bay, famosa por la película de 2000 The Beach, para permitirle recuperarse de la contaminación y otros daños causados por los turistas. Y en 2017, Indonesia declaró una «emergencia de basura» en partes de Bali». ${ }^{2}$

2. UNO Environment Programme. «Paradise lost? Travel and tourism industry takes aim at plastic pollution but more action needed», 27 de febrero de 2019, disponible en bit.ly/385UOUy. 
En situación similar se encuentran otros grandes atractivos turísticos, entre ellos, la muy amenazada Rapa Nui (Gennip, Dewitte, Garçon y Thiel, 2019).

Pero las consecuencias económicas no solo las padece un sector. La Unión Europea ha estimado que el costo por basura marina les representaría entre unos 259 y 695 millones de euros. ${ }^{3} \mathrm{El}$ manejo de los desechos plásticos está siendo tremendamente costoso, en una cuestión que en Chile también se ha advertido. ${ }^{4}$

Los desechos plásticos afectan estilos de vida y costumbres. Aun cuando no se han modelado las consecuencias en zonas colindantes a sus lugares de acopio, sí se han advertido las consecuencias en zonas costeras, en donde su población, cada año con mayor preocupación ve cómo el borde marino se encuentra plagado de esta basura, debiendo invertir grandes esfuerzos en limpieza: «En Chile ya se cumplen 11 años desde que se lleva a cabo la limpieza de playas. En 2016, se limpiaron 218 kilómetros de playas en 103 localidades desde Arica a Puerto Williams, y se retiraron 93 toneladas de residuos». De ello, se estima que «alrededor de un $80 \%$ de la basura encontrada en el mar corresponde a fuentes terrestres de origen antrópico - relativo al ser humano-y que el 90\% de los residuos en los océanos corresponde a plástico, que tarda hasta 400 años en degradarse». 5

Una de las mayores afectadas, sin embargo, es la naturaleza. Sin siquiera gozar de los beneficios del producto, día a día miles de animales están sufriendo una tortura por su causa, llevando a algunas especies a una muerte agónica. Tortugas con bombillas incrustadas en sus cuerpos, aves atrapadas, especies con deformaciones físicas luego de quedar enredadas en plástico, ciervos, peces y ballenas muertos por inanición luego de ingerir grandes volúmenes del material, son solo algunos de los crudos e impresentables episodios. Con el paso de los años, se ha reportado que las especies afectadas aumentan drásticamente, pasando de 247 contabilizadas al año 1997 a más de 690 al año 2015, de las cuales buena parte corresponden a especies incluidas en la lista roja de especies amenazadas. ${ }^{6}$

3. Parlamento Europeo, «Plásticos en el océano: datos, consecuencias y nuevas normas europeas (Infografía)», 12 de diciembre de 2018, disponible en bit.ly/3aeORaa.

4. Esta situación ya ha sido considerada en la regulación chilena. Es más, uno de los motivos que llevó a la dictación de la Ley 20.920, sobre Responsabilidad Extendida del Productor, fue el gran costo que generan los residuos: «El manejo de residuos y su disposición final generan altos costos ambientales, sociales y también un elevado costo económico para los Municipios, [...]. Un gran porcentaje de residuos son dispuestos en vertederos y microbasurales ilegales que en su mayoría se ubican en la periferia de la zona urbana, afectando principalmente a comunas de bajos ingresos e impactando negativamente sus presupuestos, debiendo estas asignar recursos económicos, equipamiento y personal para clasificar, extraer, transportar y eliminar los residuos dispuestos ilegalmente en el espacio público» (Biblioteca del Congreso Nacional, «Historia de la Ley 21.100», 3 de agosto de 2018, disponible en bit.ly/3qRbtUj).

5. Ministerio del Medio Ambiente, «Limpieza de playas 2017: Se retiran 300 kilos de residuos en Caleta Portales en Valparaíso», 4 de octubre de 2017, disponible en bit.ly/3gGVrre.

6. Un análisis y recopilación de cifras sobre los efectos del plástico en la naturaleza se puede encontrar 
La permanencia de esta clase de desechos, su aumento exponencial y difícil tratamiento deja también a las generaciones futuras particularmente vulnerables. En los océanos ya hay 5 mega islas de plástico flotando a la deriva de las corrientes que día a día aumentan su tamaño. Pero las próximas generaciones no solo tendrán que lidiar con grandes volúmenes de basura, sino que también con el manejo de sustancias tóxicas que contienen y/o trasladan algunos de estos desechos de gran durabilidad, que con su degradación devienen en microplásticos que dificultan toda esperanza de reciclaje. Como indica ONU:

Los estudios sugieren que las bolsas de plástico y los contenedores hechos de espuma de poliestireno pueden tomar hasta miles de años en descomponerse y contaminan suelo y agua. Además, con el paso del tiempo, los plásticos se dividen en fragmentos más pequeños llamados microplásticos que al ser consumidos por animales marinos pueden entrar en la cadena alimenticia humana. ${ }^{7}$

Como si fuera poco, los desechos plásticos son parte del problema del cambio climático. Los puntos de conexión son varios, no solo por la afectación y pérdida de biodiversidad, sino que están poniendo en peligro uno de los sistemas planetarios clave, los océanos. Sobre esto, se ha explicado que:

Aparte de la contaminación atmosférica, la polución química del océano [...] altera los ciclos naturales de nutrientes y/o los procesos metabólicos de especies clave en las tramas tróficas (proceso de transferencia de energía y materiales alimenticios a través de una serie de organismos), provocando entre otras cosas, problemas de eutroficación, pérdida de biodiversidad y alteración de ciclos de vida en especies marinas [...]. Los microplásticos tienen efectos en la trama trófica y pueden contribuir directamente a la pérdida de biodiversidad marina (por ejemplo, la imagen de la tortuga con una bombilla en su nariz que dio la vuelta al mundo). Peor aún, la contaminación y el cambio climático actúan de manera sinérgica, es decir, su impacto conjunto es multiplicativo. Esto implica que un organismo o un ecosistema afectado por un evento de contaminación, tendrá menos capacidad de respuesta a otras perturbaciones como las generadas por el cambio climático (Ministerio del Medio Ambiente y COP 25, 2019: 11).

Así, la relación entre desechos plásticos y cambio climático no es solo una conexión indirecta y contingente vinculada al desarrollo sustentable, la sobreexplotación de recursos y la sobrecarga -innecesaria- de ambientes. Si se considera su producción, solo el año 2006 generó emisiones de dióxido de carbono cercanas a las 2000

en SEO Birdlife y Ecoembes, Informe Libera. Impacto del abandono del plástico en la naturaleza (2019), disponible en bit.ly/3adsPF7.

7. Noticias ONU, «O nos divorciamos del plástico, o nos olvidamos del planeta», 5 de junio de 2018, disponible en bit.ly/3mg 4 lxg. 
millones de toneladas. ${ }^{8}$ En su fase de disposición, la exposición del plástico al sol emite potentes gases de efecto invernadero, ${ }^{9}$ cuestión no menor si se considera que al año 2015 ya existían en el ambiente unos 380 millones de toneladas, las que van en aumento cada año. En términos de $\mathrm{CO}_{2}$, «reciclar 1 millón de toneladas de plástico, equivale a retirar 1 millón de automóviles de las carreteras». ${ }^{10}$ En contraste, su incineración (que al año 2015 alcanzó a un 12\% del plástico desechado) genera monóxido de carbono entre otros metales pesados.

Estas y muchas otras consideraciones han puesto la preocupación sobre el material a nivel mundial, ya que de no hacerse nada, se mantendrá un aumento exponencial. ${ }^{11}$ Uno de los primeros pasos que urge considerar es la prevención de su rápido desecho que pueden permanecer por miles de años en el planeta. En esto los plásticos de un solo uso (botellas, envoltorios, bombillas, entre muchos otros) son una puerta de entrada en la gestión de los efectos adversos, cuestión que ha gatillado la necesidad regulatoria de restringir su uso como se pasa a analizar.

\section{Regulación internacional y comparada de los desechos plásticos}

Aun cuando no existe un tratado que expresamente se refiera al problema global de los desechos plásticos, distintos actores y estados han adoptado y/o promovido una serie de regulaciones en torno al plástico, encontrando algunas referencias ya muy tempranas. En efecto, durante el curso del siglo XX, el plástico fue rápidamente normado, particularmente en cuanto a su contacto y exposición con alimentos, por su toxicidad y también respecto sus desechos. ${ }^{12}$

Solo con efectos sistematizadores, si se analizan en los actores, se puede distinguir una reacción a nivel internacional, el intento comunitario de la Unión Europea,

8. France 24, «Parlamento Europeo prohíbe los plásticos de un solo uso a partir de 2021». 28 de marzo de 2019, disponible en bit.ly/2Wewry4.

9. Sobre las emisiones de gases con efecto invernadero, se puede buscar información en ONU Programa para el medio ambiente, «Un problema doble: el plástico también emite potentes gases de efecto invernadero», 24 de agosto de 2018, disponible en bit.ly/30Iy976.

10. Parlamento Europeo, «Plásticos en el océano: datos, consecuencias y nuevas normas europeas (infografía)», 12 de diciembre de 2018, disponible en bit.ly/3aeORaa.

11. Se ha rescatado que, «en 2020 el ritmo de producción de plásticos habrá aumentado un $900 \%$ con respecto a niveles de 1980 (más de 500 millones de toneladas anuales). La mitad de este incremento se producirá tan solo en la última década» (Greenpeace, Plásticos en los océanos. Datos, comparativas e impactos, 2016, disponible en bit.ly/3ngkOmm).

12. Por ejemplo, el año 1992, el Mercosur indicaba: «es necesario compatibilizar las legislaciones vigentes en los Estados Parte del Mercosur relacionadas con los envases y equipamientos en contacto con alimentos, que tengan en cuenta primordialmente la protección de la salud humana, y también los aspectos económicos y tecnológicos» (Mercosur, 1992: 1). 
regulaciones estatales, en lo que se resaltará el caso de China, por su repercusión; y, finalmente, esfuerzos más locales.

\section{Compromisos internacionales}

Existen algunos tratados desde los cuales se puede construir una referencia al manejo de esta clase de desechos. Por lo pronto, como uno de los puntos de referencia, interesa destacar el Protocolo de Kyoto de la Convención Marco de las Naciones Unidas sobre el Cambio Climático, desde el cual es posible extraer elementos orientativos sobre la regulación de los desechos plásticos, bajo la meta de la prevención de gases con efecto invernadero por la generación y gestión de residuos.

Este protocolo, en particular sobre los desechos, contempla como uno de sus objetivos que los estados limiten y/o reduzcan emisiones mediante una recuperación y optimización de la utilización en la gestión de los desechos (artículo 1). En su anexo A, bajo la categoría «desechos», también es posible encontrar una referencia sobre la necesidad de implementar medidas preventivas y adaptativas para la gestión de residuos, de modo que no se transforme en una fuente de emisión.

Desde esta y otras consideraciones, la ONU ha profundizado por medio de distintos instrumentos la necesidad de regular y limitar los plásticos de un solo uso. Así, publica el año 2018 una completa guía sobre «la experiencia de países que han impuesto prohibiciones y regulaciones sobre los plásticos de un solo uso», para que los diseñadores de políticas puedan fácilmente advertir las distintas medidas posibles, sus resultados y sus niveles de eficiencia (ONU Medio Ambiente, 2018).

Cabe señalar que el informe reitera que la regulación debe considerar todo el ciclo de vida del plástico (producción, consumo, recuperación y desecho), haciendo presente que lo esencial es promover mejoras y gestión de residuos, generar alternativas ecológicas y concientizar a las personas por un uso irresponsable para una reducción voluntaria. Asimismo, entrega una hoja de ruta para diseñadores de políticas con diez pasos detallados que tienen por objeto orientar a los gobiernos a tomar medidas en contra de los desechos plásticos.

En resumen, indica que se debe considerar i) cuáles son las condiciones iniciales, esto es los plásticos problemáticos, sus volúmenes, magnitud, impactos y origen; ii) evaluar las alternativas que más rendimiento pueden tener (compromisos voluntarios de ciertos actores, regulaciones económicas, una combinación); iii) investigar los impactos de la o las opciones consideradas; iv) involucrar a los interesados; v) desarrollar programas de concientización y comunicación de medidas; vi) promover alternativas a plásticos regulados; vii) promover en las industrias un cumplimiento, con políticas de transición y/o reembolso; viii) asegurar ganancias para la industria del reciclaje, minimizar residuos, y financiar la concientización; ix) velar por el cum- 
plimiento de las políticas, promoviendo roles y responsabilidades; y finalmente $\mathrm{x}$ ) monitorear y ajustar las alternativas escogidas.

A este completo informe, agregó luego una segunda hoja informativa titulada Prohibición de plásticos de un solo uso: conjunto de directrices para la sostenibilidad. Hoja informativa para diseñadores de políticas ${ }^{13}$ la cual se concentra en los plásticos de rápido desecho, enumera los más problemáticos, llama a priorizar la reducción de bolsas plásticas y productos de espuma de poliestireno e indica 5 acciones prioritarias para minimizar el impacto de los plásticos de un solo uso: i) mejorar los sistemas de residuos; ii) promover alternativas ecológicas; iii) educar a los consumidores; iv) promover estrategias de reducción voluntaria de todos los actores; y v) prohibir, gravar con impuestos o ambos, el uso y venta de plásticos de un solo uso.

También es preciso indicar que la Cuarta Asamblea de las Naciones Unidas para el Medio Ambiente, celebrada en Kenia en marzo de 2019 concluyó con una importante declaración de los ministros asistentes, que manifestaron una profunda preocupación porque el planeta sigue aumentando significativamente sus niveles de contaminación, lo que genera efectos adversos sobre el cambio climático, una rápida pérdida de biodiversidad y degradación del medio ambiente, todo ello, pese a que existen diferentes alternativas. Ante la preocupación por este escenario, se indicó como uno de los compromisos centrales, reducir al año 2030 significativamente los plásticos de un solo uso y eliminar aquellas prácticas no sostenibles relacionadas con el material, para lo que se propuso trabajar con privados en la búsqueda de soluciones ecológicas. ${ }^{14}$

\section{Una respuesta comunitaria: las propuestas de la Unión Europea}

Al igual que en materia internacional, desde un bueno tiempo la Unión Europea manifestó su preocupación por la magnitud y consecuencias de los desechos plásticos. Uno de los puntos de partida que se puede considerar fue la Directiva 94/62/CE del Parlamento Europeo y del Consejo de 20 de diciembre de 1994, relativa a los envases y residuos de envases, que tenía por objeto dar reglas comunes para «armonizar las diversas medidas nacionales sobre gestión de envases y residuos de envases» (Directiva 94/62/CE:1).

Por esta Directiva ya desde el año 1994 se reconoce que el crecimiento económico debe ser sostenible y compatible con el medio ambiente y que los envases por razones de higiene, seguridad y salubridad tienen una función comunitaria esencial. Promue-

13. Disponible en bit.ly/2JYrrv2.

14. ONU Programa para el medio ambiente, «Líderes mundiales se reúnen en el máximo órgano ambiental de la ONU para impulsar economías más», 10 de marzo de 2019, disponible en https://bit. ly/3hiDsYv. 
ve el retorno de envases usados e indica que «el reciclado deberá constituir una parte importante de la valorización, con el propósito fundamental de reducir el consumo de energía y de materias primas básicas y la eliminación final de residuos» (Directiva 94/62/CE: 1-2).

Así, en esa época, la preocupación seguía centrada en la toxicidad de los productos:

Es necesario, como primer paso para reducir la toxicidad de los residuos de envases, evitar la adición de los metales pesados nocivos en la fabricación de envases, o controlar que tales elementos no vayan a parar al medio ambiente, con las oportunas excepciones en casos concretos que han de ser establecidos por la Comisión de conformidad con un procedimiento de Comité (Directiva 94/62/CE: 2).

No obstante, advertía que «la separación de los residuos en el origen es fundamental para conseguir un alto nivel de reciclado y para evitar problemas de salud y de seguridad a las personas encargadas de recoger y tratar los residuos de envases» (Directiva 94/62/CE: 2).

Además, ya en el año 94 hacía un llamado a la responsabilidad compartida y extendida:

Todos aquellos que intervienen en la producción, el uso, la importación y la distribución de envases y productos envasados adquieran mayor conciencia del grado en que dichos envases se transforman en residuos, y de que acepten, de conformidad con el principio de que «quien contamina paga», la responsabilidad de dichos residuos (Directiva 94/62/CE: 3).

En el año 2008 con la Directiva 2008/98/CE del Parlamento Europeo y del Consejo, ante el aumento del problema, se intentan retomar medidas:

El primer objetivo de cualquier política en materia de residuos debe ser reducir al mínimo los efectos negativos de la generación y la gestión de los residuos para la salud humana y el medio ambiente. La política en materia de residuos debe tener también por objeto reducir el uso de recursos y favorecer la aplicación práctica de la jerarquía de residuos (Directiva 2008/98/CE: 2 ).

Con ello, se posiciona a la prevención de residuos como el punto de partida.

Impuso objetivos, como que al año 2020 se aumente en un 50\% la reutilización y reciclado, e instó a que los estados realizaran una recogida separada de los materiales (artículo 11 de la directiva citada).

Con todo, por la escalada del problema de los desechos plásticos, siete años después, se promulgó la Directiva (UE) 2015/720 del Parlamento Europeo y del Consejo de 29 de abril de 2015, que se refiere a la reducción del consumo de bolsas de plástico ligeras, complementada tres años después con la Directiva (UE) 2018/851 del Parlamento Europeo y del Consejo de 30 de mayo de 2018, según la cual: 
La gestión de residuos en la Unión debe mejorarse y transformarse en una gestión sostenible de las materias con miras a proteger, preservar y mejorar la calidad del medio ambiente, así como a proteger la salud humana, garantizar la utilización prudente, eficiente y racional de los recursos naturales (Directiva 2018/851: 1).

Así se advierte un nuevo elemento: la motivación pasa a ser también económica: mejorar el uso de la energía, reducir la dependencia de materia prima, desarrollar un mercado competitivo, promover una economía circular que impulse la creación de actividades bajo una producción y consumo sostenible. Es más, se rescata que la sola gestión eficiente, permitiría un ahorro considerable a empresas, gobiernos y consumidores, todo ello reduciendo gases de efecto invernadero.

La nueva normativa mantiene y reitera la necesidad de una recolección segregada de plásticos, para maximizar la reutilización y reciclaje, ya que cuando distintos plásticos se mezclan, su posibilidad de utilización es muy baja. Agrega que los estados miembros tendrán que adoptar medidas «para garantizar que los productores asuman la responsabilidad financiera y organizativa de la gestión de la fase de residuo del ciclo de vida de un producto» (Directiva 2018/851: 2).

Enfatiza la prevención y la generación de productos con mayor vida útil:

Los Estados miembros adoptarán medidas para prevenir la generación de residuos. Como mínimo, esas medidas: a) promoverán y apoyarán los modelos de producción y de consumo sostenibles, b) fomentarán el diseño, la fabricación y el uso de productos que sea eficientes en el uso de recursos, duraderos (también en términos de vida útil y ausencia de obsolescencia programada), reparables, reutilizables y actualizables (Directiva 2018/851: 18).

Luego, la Directiva (UE) 2018/852 del Parlamento Europeo y del Consejo de 30 de mayo de 2018, enfatiza en la necesidad de profundizar en envases y residuos de envases reutilizables, que no solo descansen en un eventual y costoso reciclaje. ${ }^{15}$ Profundiza en la necesidad de la prevención y la instalación de una economía circular para Europa que dependa menos de materias importadas:

El fomento de una bioeconomía sostenible puede contribuir a reducir la dependencia de la Unión con respecto a las materias primas importadas. Los envases reciclables de origen biológico y los envases biodegradables compostables pueden representar una oportunidad para promover la fabricación de envases a partir de fuentes renovables (Directiva 2018/852: 7).

Vuelve a poner en el panorama la responsabilidad del productor, ya que es él

15. Señala la Directiva, «resulta necesario que los Estados miembros adopten medidas adecuadas para fomentar un aumento de la proporción de envases reutilizables comercializados y la reutilización de los envases» (Unión Europea, 2018, Directiva 2018/852: 5). 
quien determina la distribución de su producto, sin dar espacio a los consumidores e impone nuevamente metas medibles: al 2025 aumentar un 50\% el peso de los plásticos reciclados, al 2030 un 55\% del peso de plásticos reciclados; diseños que permitan su reutilización y valoración y define las condiciones de un plástico biodegradable, descartando alternativas como envases oxodegradables, por su imposibilidad de tratamiento y la generación de microplásticos (Directiva 2018/852).

Aun con toda esta regulación, el 2019 la Unión Europea intenta extremar recursos con la Directiva (UE) 2019/904 del Parlamento Europeo y del Consejo 5 de junio de 2019 para «prevenir y reducir el impacto de determinados productos de plástico en el medio ambiente, en particular el medio acuático, y en la salud humana, así como fomentar la transición a una economía circular» (artículo 1 de la Directiva 2019/04).

En este nivel, ahora apunta directamente a los plásticos de un solo uso con:

Las medidas necesarias para lograr una reducción ambiciosa y sostenida del consumo de los productos de plástico de un solo uso [...]. De aquí a 2026, dichas medidas tendrán que lograr una reducción cuantitativa medible del consumo. [Por ello] A más tardar el 3 de julio de 2021, los Estados miembros prepararán una descripción de todas las medidas que hayan adoptado (Directiva 2019/904: 9).

A diferencia de otras directivas son plásticos específicos: plásticos de un solo uso, productos fabricados con plástico oxodegradable, artes de pesca que contienen plástico. Y:

A fin de determinar si un recipiente para alimentos ha de considerarse producto de plástico de un solo uso a los efectos de la presente Directiva, además de los criterios enumerados en el anexo en relación con los recipientes para alimentos, desempeñará un papel decisivo su tendencia a convertirse en basura dispersa, debido a su volumen o tamaño, en particular las porciones individuales (Directiva 2019/904: 14).

Según el tipo de producto diferenciado establece medidas concretas: «prohibirán la introducción en el mercado de los productos de plástico de un solo uso enumerados en la parte B del anexo y de los productos fabricados con plástico oxodegradable» (Directiva 2019/904: 10); e impone medidas sobre el diseño de productos enlistados la gestión de información, etiquetados, remisión de datos a la Unión Europea y medidas para incentivar un comportamiento responsable.

Finalmente, queda a discreción de los estados las sanciones, lo que muestra la amplia y sostenida regulación europea en la materia, que de manera sostenida en el tiempo ha ido adoptado diversas medidas, desde la exigencia de prevención, la responsabilidad del productor por intentar la recuperación del material, la proscripción de cierto tipo de materiales (oxodegradables), medidas de ecodiseño, reutilización, reducción de envases y las prohibiciones de ciertos plásticos de un solo uso. 


\section{Reacción estatal: la decisión china y el gran impacto en el mercado mundial del desecho plástico}

La preocupación de los estados sobre la materia parece ser una tónica mundial. Muestra de ello, es que «el 10 de mayo más de 180 naciones acordaron en Ginebra agregar los desechos de plástico mixto al Convenio de Basilea, el tratado que controla el movimiento internacional de desechos peligrosos». ${ }^{16} \mathrm{~A}$ su vez, informes de la ONU concluyen que al año 2018, al menos 27 países cuentan con una ley o tipo de regulación que se refiera a los plásticos de un solo uso, a un material determinado o a niveles de producción de este material. Esta cifra no considera las naciones que han regulado las bolsas plásticas, en cuyo caso la cifra aumenta a 127 países. Junto con esto se encontró que al menos 63 países tienen alguna regulación sobre la responsabilidad extendida del productor (United Nation Environment Program, 2018).

Pese a ello, existen ciertos casos icónicos por su amplia repercusión. Tal es el caso de China que históricamente ha sido apuntado como uno de los grandes productores y contaminantes de plástico. ${ }^{17}$ La potencia asiática en su proceso de industrialización en la década de los ochenta enfrentó una crisis de materias primas que solucionó importando residuos sólidos de distintos países (México, Estados Unidos y principalmente Europa). Para transformar esos desechos en valor económico desarrolló una fuerte industria de reciclado estructurada en gestores de residuos locales. Así, la industria del reciclaje fue especializándose en distintos materiales: chatarra electrónica, textiles, plásticos, residuos minerales (hierro, metales, cobre), identificándose barrios y provincias según el material reciclado. ${ }^{18}$

No obstante, esta compra masiva de basura se detuvo súbitamente, pues a inicios del año 2018:

[Y] con el objetivo de garantizar la sostenibilidad medioambiental del país, [...] entró en vigor la prohibición de introducir en China 24 tipos de residuos agrupados en cuatro categorías: plásticos domésticos, papel sin clasificar, varios tipos de escoria de minas y desechos textiles. ${ }^{19}$

16. ONU, Programa para el Medio Ambiente, «Gobiernos acuerdan decisiones históricas para erradicar o regular desechos y productos químicos», 12 de mayo de 2019, disponible en bit.ly/2IL4BGJ.

17. «China es el mayor productor de plástico del mundo. Se estima que en 2010 solo cinco países asiáticos -China, Indonesia, Filipinas, Vietnam y Sri Lanka- generaron la mitad de la basura plástica mal gestionada de todo el planeta». National Geographic, «Yiwu, la capital internacional del plástico cotidiano», 16 de junio de 2020, disponible en bit.ly/3qUvJUP.

18. Para ver mayor información sobre el sistema de reciclaje en China: Biblioteca Nacional del Congreso, «El sistema de reciclaje en China que potencia el rol de los gestores de residuos», 18 de mayo de 2018, disponible en bit.ly/3473boA.

19. Agencia EFE, «China amplía veto a «basura extranjera» y prohíbe otros 32 tipos de desechos», 19 de noviembre de 2018, disponible en bit.ly/2W8oviu. 
Fueron las propias autoridades del país asiático las que salieron a aclarar que, con su propia producción de desechos, les es suficiente para cubrir sus necesidades productivas. ${ }^{20}$ Incluso, precisaron que «la economía se ve favorecida, porque los países podían enviar desechos reciclables a China en los mismos contenedores utilizados para importar productos manufacturados» ${ }^{21}$

Por barreras idiomáticas se desconoce el detalle de la regulación interna de los plásticos en la potencia asiática. Pero China, además de haber impulsado una industria fuerte del reciclaje, en base a un sistema de incentivos, impulsó la creación de zonas de recepción de residuos de forma tal que cada vez que una persona reciclaba, acumulaba una especie de puntos que luego podían ser canjeados. Junto con esto, también contempló impuestos, prohibiciones y sanciones. ${ }^{22}$

El sistema de incentivos, la prohibición de importación de plásticos, la generación de una economía circular y la industria instalada de reciclaje, junto con ser una muestra de que los plásticos desechados están siendo regulados por China, muestra también una preocupación por el exceso de este tipo de residuos. De hecho, eso fue lo que justificó la medida, desechos difíciles de manejar, que se entremezclan con materias primas e impiden o encarecen su tratamiento, siendo incluso, alguno de ellos tóxicos o peligrosos poniendo en riesgo la vida de las personas.

Con esta decisión, el desafío (que quizás explica la gran preocupación europea como se vio) comenzó a ser el manejo interno de cada estado por residuos que ya no serán recibidos en China. Los datos muestran que «China ha importado el 45 por ciento de los residuos mundiales totales desde 1992. (Si se añade Hong Kong, que volvió a la soberanía de China en 1997 tras 156 años como colonia británica, el porcentaje de China pasa a ser el 70 por ciento)». ${ }^{23}$

Así, luego de la prohibición:

Fardos de desechos se acumularon en California, Reino Unido y Australia, entre otros países, mientras las naciones exportadoras buscaban nuevos exportadores. En todo el Sudeste Asiático, los recicladores que operan en Indonesia, Tailandia, Vietnam y Malasia compraron residuos, pero enseguida se vieron abrumados al recibir la gigantesca cantidad que China había absorbido. ${ }^{24}$

20. BBC News, «Por qué China quiere dejar de ser el basurero del mundo y cómo eso afecta al resto de los países», 8 de enero de 2018, disponible en bbc.in/2IJVR 3 E.

21. Catherine Early, «China renews clampdown on waste imports», China Dialogue, 31 de julio de 2017, disponible en bit.ly/386pGUW.

22. Wang Chen, «Should Chinese citizens be paid to recycle?», China Dialogue, 10 de enero de 2019, disponible en bit.ly/2IOPPim.

23. Laura Parker, «El veto de China a la importación de basura desplaza la crisis de residuos al Sudeste Asiático», National Geographic, 19 de noviembre de 2018, disponible en bit.ly/349uQhC.

24. Laura Parker, «El veto de China a la importación de basura desplaza la crisis de residuos al Sudeste Asiático», National Geographic, 19 de noviembre de 2018, disponible en bit.ly/349uQhC. 


\section{Esfuerzos locales: algunos estados, provincias y comunas también han tomado medidas de regulación de los residuos plásticos}

A estos esfuerzos estatales también se suman esfuerzos locales. Un caso llamativo fue el Estado de Nueva York que, en abril del año 2019 decidió como primera medida prohibir a las agencias públicas de la ciudad, comprar plásticos de un solo uso. En aquella oportunidad, su alcalde dio un plazo de 120 días para que todos los organismos aplicaran e hicieran efectiva la medida, y prometió avanzar prontamente en una segunda etapa, estableciendo una prohibición que alcance directamente a privados. ${ }^{25}$

En el contexto latinoamericano, también hay avances presentes en distinto nivel. Por ejemplo, la ciudad de Buenos Aires, el 22 de mayo de 2019, prohibió la utilización, entrega y venta de bombillas plásticas de un solo uso (Ministerio del Medio y Ambiente y Espacio Público Argentino, 2019). En una dirección similar se encuentra la ciudad de Guayaquil, mientras que en Galápagos se pretende hacer efectiva una normativa que restringe los plásticos de un solo uso en el archipiélago, lo que comprende grupos de productos como bombillas, botellas plásticas o polietileno expandido con aire.

Mientras en Colombia se encuentra en marcha una discusión legislativa que busca prohibir plásticos de un solo uso, en Perú ya se dictó una ley especial: Ley 30.884, que regula el consumo de bienes de plástico de un solo uso que generan riesgo para la salud pública y/o el ambiente. Enfocada en alimentos y bebidas de consumo humano, regula plástico de un solo uso, plásticos no reutilizables y poliestireno expandido. La técnica también se centra en un grupo de productos, tales como bolsas, bombillas, envases para alimentos y bebidas. Establece distintos plazos según el área o zona de prohibición; un registro de fabricantes, importadores y distribuidores; la carga de una educación ciudadana ambiental; la obligación del uso de material reciclado para botellas; y la inclusión de un certificado de biodegradabilidad o equivalente.

\section{Los desechos plásticos y los plásticos de un solo uso ante el ordenamiento jurídico chileno}

Una respuesta rápida, pero que puede llevar a equívocos, va por afirmar que en Chile no existe una respuesta jurídica al problema de los desechos plásticos y en particular a los plásticos de un solo uso. Aun cuando no exista una normativa a nivel nacional que de forme expresa regule la materia, es posible modelar una respuesta y análisis en torno al problema que están desencadenando aquellos plásticos que, con muy poco tiempo de uso, son rápidamente desechados.

25. Información sobre la medida y las declaraciones realizadas, disponible en: NYC, «Mayor de Blasio Signs Executive Order to End City Reliance on Single-Use Plastic», 11 de abril de 2019, disponible en on.nyc.gov/3mgeV7x. 
Para explorar la respuesta nacional, se realizará una aproximación constitucional a la pregunta y la forma en la que esos preceptos pueden orientar la actuación de los incumbentes; una somera revisión a algunos compromisos internacionales suscritos, los cuales también tienen algún criterio orientativo en torno al uso que se pueda dar al material; una aproximación desde la Ley de Responsabilidad Extendida del Productor (Ley REP); ciertos esfuerzos municipales, teniendo como punto de referencia el caso de la comuna de Providencia; para finalizar con una breve revisión a la discusión legislativa actual.

\section{Una aproximación constitucional a la prohibición de plásticos de un solo uso}

Si se olvida por segundos la pregunta por el material en sí mismo (que es el objeto de la regulación) y se ven en sus efectos (rescatados en el primer apartado), la pregunta se convierte por la identificación de derechos involucrados y la compatibilización de distintos intereses. Para esto, el lenguaje constitucional, de la mano de la teoría los derechos fundamentales, entrega una interesante aproximación que reconoce la libertad de un productor, distribuidor y consumidor para emplear un material, pero también la afectación a la vida y salud de generaciones presentes y futuras, el respeto al medio ambiente, la protección de otros rubros económicos y la utilización eficiente y focalizada de recursos públicos.

En esto, la teoría indica que los derechos fundamentales pueden ser restringidos, ${ }^{26}$ en la medida que no se esté ante un acto abusivo, arbitrario o desproporcional. ${ }^{27}$ Así, desde el principio de proporcionalidad se ha sostenido que se debe analizar si el fin perseguido es legítimo, si la medida es idónea, necesaria y si no es excesivamente gravosa.

Volviendo ahora sobre el plástico, el fin perseguido no es su prohibición sino que proteger la salud de las personas, la naturaleza, otras actividades económicas y la focalización de recursos públicos. La idoneidad puede ser considerada desde la experiencia comparada, en donde la prohibición de ciertos plásticos innecesarios es una estrategia

26. En la misma teoría constitucional, otro punto que enfoca la discusión se traduce en el título de intervención, que habilitaría al Estado a restringir un producto. Ante esto, nuestro ordenamiento constitucional también da variadas respuestas: el respeto y promoción de derechos fundamentales, la cláusula ambiental del artículo 19 número 8 y la limitación de la propiedad por su función social son alguna de ellas.

27. Sobre esto el Tribunal Constitucional ha fallado que las restricciones son jurídicamente permitidas, en la medida que sean razonables, no arbitrarias y proporcionales: «debe ser razonable, no arbitraria, sirviendo como referencia del juicio de razonabilidad la concurrencia del principio de proporcionalidad, determinado por la relación coherente entre los medios utilizados y los fines legítimos perseguidos» (Tribunal Constitucional de Chile, Sentencia de término, dictada en causa rol 541-06, 13 de julio de 2006). 
común y en aumento, que evita la generación y uso abusivo del material, en circunstancias en las que han ponderado otras vías (complementarias y/o sustitutivas). Más aun, las evaluaciones y estudios de la experiencia recopilada por ONU, también rescatadas previamente, confirman lo necesario y apto de la medida. Finalmente, se debe también poner en su justa ponderación la propuesta, pues no se trata de una medida drástica que prohíbe toda una industria o que proscribe por completo el uso del material.

De este modo, desde la lógica constitucional, parece completamente razonable que se avance en esta prohibición. Cada uno de los bienes jurídicos identificados tiene una traducción constitucional que debe ser ponderada, pudiendo ser construida esta medida como una forma de protección de derechos fundamentales, uno de los deberes impuesto como sociedad.

Más aún, no se debe perder de vista que el ordenamiento chileno, desde siempre ha admitido la restricción de actividades o ciertos productos, en atención a otros derechos o intereses. Basta recordar los artículos 1463 a 1466 del Código Civil que, por motivos como la moral o el orden público económico, impiden la suscripción de ciertos actos de cotidiana aplicación. Por lo demás, la medida es únicamente para ciertos usos del plástico, típicamente envases que mediante su rápido desecho se encuentran contaminando el ambiente desproporcionalmente y afectando cíclicamente otros derechos.

Desde este marco conceptual, la prohibición a cierto tipo de plásticos no parece una anomalía, ni mucho menos una exageración. Por el contrario, esta aproximación indica que su restricción es un mecanismo de resguardo y protección de derechos constitucionales, de modo que, aun sin ley o normativa específica, se puede ver que la Constitución y ponderación de derechos impulsan a todos los actores (gobierno, productores, distribuidores y consumidores) para utilizar el material de forma razonable evitando volúmenes actuales de desechos.

\section{Los Tratados suscritos que deben ser considerados}

Un segundo paso del análisis nacional es considerar los criterios sobre los cuales como estado estamos obligados internacionalmente a respetar. Para ello, sin referirse expresamente a los plásticos, ya en 1972 se acordó el Convenio sobre la prevención de la contaminación del mar por vertimiento de desechos y otras materias, que establece el deber de los estados de proteger el medio marino de toda fuente de contaminación, ${ }^{28}$ lo que comprende las grandes cantidades de desecho plástico que

28. En este sentido, el artículo 2 del Convenio sobre la prevención de la contaminación del mar por vertimiento de desechos y otras materias indica: «Artículo 2. Las Partes Contratantes, individual y colectivamente, protegerán y preservarán el medio marino contra todas las fuentes de contaminación y adoptarán medidas eficaces, según su capacidad científica, técnica y económica, para prevenir, reducir y, cuando sea factible, eliminar la contaminación causada por el vertimiento o la incineración en el mar de desechos u otras materias. Cuando proceda, las Partes Contratantes armonizarán sus políticas a este respecto». 
están llegando al océano. ${ }^{29}$

Por su parte, la Convención Marco de las Naciones Unidas sobre el Cambio Climático, de 9 de mayo de 1992, que daría origen a la COP, establece una conexión entre el cambio climático y el ciclo de vida de los desechos, desde su generación hasta su extinción. En ello, entre otras, se asume como compromiso adoptar las prácticas que reduzcan emisiones por desechos, cuestión relevante pues no solo la generación de plásticos produce gases de efecto invernadero, sino que todo su ciclo (distribución y desecho)..$^{30}$

De igual modo, se pueden rescatar elementos de la Declaración de Río sobre el Medio Ambiente y el Desarrollo del año 1992 que estableció una serie de principios en torno a un desarrollo económico respetuoso de los ecosistemas, que aborde en mejor forma los problemas de la degradación ambiental (principios 7 y 12). Nuevamente, sin una referencia directa a los plásticos, el principio 8 contiene una directriz tremendamente relevante sobre el sistema de uso sostenible que se tiene que dar al material:

Principio 8. Para alcanzar el desarrollo sostenible y una mejor calidad de vida para todas las personas, los Estados deberían reducir y eliminar las modalidades de producción y consumo insostenibles y fomentar políticas demográficas apropiadas.

Esto es particularmente relevante considerando el aumento exponencial y constante de los plásticos de un solo uso que, pese a una duración de cientos o miles de años, son rápidamente consumidos, desechados y sustituidos por otro material que ingresa al mismo ciclo de uso, desecho y sustitución de otro igual.

La Declaración de Río también permite advertir otro elemento. No se debe dejar pasar los problemas internacionales que generan los desechos plásticos que, al ingresar a cursos de agua quedan sin control de su paradero final, en lo que parece ser una infracción manifiesta al principio 14 de la Declaración:

Principio 14. Los Estados deberían cooperar efectivamente para desalentar o evitar la reubicación y la transferencia a otros Estados de cualesquiera actividades y

29. Es más, el artículo 15 del tratado contempla una cláusula de responsabilidad acompañada de una serie de reglas sobre solución de controversias por incumplimiento del pacto: «Artículo 15. De conformidad con los principios del derecho internacional relativos a la responsabilidad de los Estados por los daños causados al medio ambiente de otros Estados o a cualquier otra zona del medio ambiente, las Partes Contratantes se comprometen a elaborar procedimientos relativos a la responsabilidad por vertimiento o incineración en el mar de desechos u otras materias».

30. Sobre esto, el artículo 4 de la Convención Marco indica: «Artículo 4. 1. C. Promover y apoyar con su cooperación el desarrollo, la aplicación y la difusión, incluida la transferencia, de tecnologías, prácticas y procesos que controlen, reduzcan o prevengan las emisiones antropógenas de gases de efecto invernadero no controlados por el Protocolo de Montreal en todos los sectores pertinentes, entre ellos la energía, el transporte, la industria, la agricultura, la silvicultura y la gestión de desechos». 
sustancias que causen degradación ambiental grave o se consideren nocivas para la salud humana.

En una misma dirección se encuentra el «Programa 21», por el cual Chile se comprometió a la reducción de desechos, el aumento de reutilización y reciclado, uso racional y tratamiento ecológico, todo lo que pone en duda y contraria el uso actual del plástico:

$21.5[\ldots]$.

a) Reducción al mínimo de los desechos;

b) Aumento al máximo de la reutilización y el reciclado ecológicamente racionales de los desechos;

c) Promoción de la eliminación y el tratamiento ecológicamente racionales de los desechos;

d) Ampliación del alcance de los servicios que se ocupan de los desechos.

De este modo, incluso obviando el análisis constitucional, como compromisos internacionales hace más de 30 años que obligamos, de buena fe, a reducir la generación de desechos, consumir productos de forma responsable y sostenible, promover la reutilización y no dañar océanos y otros estados con contaminantes, observaciones que exigen replantear (a todos los actores) los plásticos de un solo uso y en general el problema de los desechos plásticos.

\section{La respuesta desde el estándar legal: ley de responsabilidad extendida del productor y los desechos plásticos}

El año 2018 se promulgó y publicó la Ley 21.100 que prohíbe la entrega de bolsas plásticas de comercio en todo el territorio nacional, marcando un hito en torno a la prevención de los desechos plásticos. Con todo, varios municipios de forma precedente ya habían dictado ordenanzas comunales en un mismo sentido, pues la espera de esta ley fue larga: el proyecto ingresó el 10 de octubre del año 2013, pese a contar con el patrocinio político transversal de todos los sectores y solo se promulgó el 2018.

Esta iniciativa se fundamenta entre otros, en que:

Los plásticos y los materiales sintéticos son los dos tipos de basura marina más común y son responsables de la mayor parte de los problemas que sufren los animales y aves marinas. [...]. Los plásticos están también presentes en las playas, desde las regiones más pobladas hasta las costas de islas remotas y deshabitadas..$^{31}$

Este mismo fundamento que se dio para prohibir las bolsas plásticas plantea la

31. Biblioteca del Congreso Nacional, «Historia de la Ley 21.100», 3 de agosto de 2018, disponible en bit.ly/3qRbtUj. 
pregunta por la suficiencia de la medida para combatir la gran cantidad de desechos plásticos que se generan en Chile: unas 990 mil toneladas anuales..$^{32}$

En esta aproximación, la Ley 20.920 sobre Responsabilidad Extendida del Productor (en adelante Ley REP), publicada el 1 de junio del año 2016, abordó el problema de los residuos en el país y si bien no se refiere directamente a los plásticos, al establecer políticas de manejo para los residuos en general, sus disposiciones cubren a esta clase de desechos. Es más, su mensaje hizo presente los problemas identificados: el manejo de residuos y su disposición final generan altos costos ambientales, sociales y también un elevado costo económico para los municipios.

Esta ley establece una serie de directrices. En primer lugar, la «jerarquía en el manejo de recursos», que considera como primera alternativa «la prevención en la generación de residuos, luego la reutilización, el reciclaje de los mismos o de uno o más de sus componentes y la valorización energética de los residuos, total o parcial, dejando como última alternativa su eliminación» (artículo 2, letra d).

Junto con ello contempla la «responsabilidad del generador de un residuo», según la cual «es responsable de este, desde su generación hasta su valorización o eliminación» (artículo 2, letra i), de forma que internalicen los costos para que no sean soportados por el medio ambiente y/o los municipios.

Buena parte de los PUSU - envases y embalajes - integran la categoría de producto prioritario, con lo que sus productores «son responsables de la organización y financiamiento de la gestión de los residuos» de conformidad con los reglamentos que se dicten en la materia por el Ministerio del Medio Ambiente, de conformidad con esta ley. Además, de ello, están obligados a ingresar a un registro público, realizar declaraciones anuales y -entre otras- se les podrá requerir información sobre la cantidad de productos comercializados, las actividades y cantidades de recolección valorización y eliminación (artículos 10 y siguientes de la ley comentada).

Entre otras, la Ley REP promueve un sistema de integración de recolectores y formas de disposición y reciclaje que, complementa con un mandato a los municipios, quienes «deberán incorporar en sus ordenanzas municipales la obligación de separar los residuos [...] cuando así lo determine el decreto supremo» (artículo 30, letra d). Para cerrar el ciclo, impone a los distribuidores y comercializadores, la carga de «aceptar sin costo la entrega de los residuos de productos prioritarios» (artículo 33, numeral 2).

También se puede encontrar en esta ley (artículo 4) las principales directrices que se han implementado en otros países para el manejo de residuos plásticos: productos que pueden ser evitados, la necesidad de mejorar su ecodiseño; certificaciones, rótulos y etiquetas, de forma que se permita conocer si es reutilizable, reciclable o

32. País Circular, «Desafío para la Ley REP: En Chile se recicla el $8 \%$ del plástico, y solo un $17 \%$ de ello proviene de los hogares», 27 de marzo de 2019, disponible en bit.ly/3aFwage. 
desechable; que se establezca un sistema de depósito y reembolso (tal como ocurre con los denominados envases retornables de bebidas, de forma que se amplíe esta práctica). La misma norma contempla que como resultado de la etiquetación y la operación de un sistema de depósito y reembolso, se instauren mecanismos para una recolección selectiva de productos.

Establece además que estas medidas se tomarán mediante Decreto Supremo dictado por el Ministerio del Medio Ambiente, el cual contendrá un proceso de participación (consulta pública), consulta a organismos públicos y privados involucrados, debiendo ser sometido ante el Consejo de Ministros para la Sustentabilidad. De este modo, hoy, la regulación de los residuos plásticos no necesariamente descansa en el Congreso, sino que el Ejecutivo se encuentra completamente habilitado para tomar medidas regulatorias.

Finalmente, no se puede dejar de observar que, aun cuando falte la dictación de los reglamentos respectivos, al menos desde el año 2016 con la publicación de la ley, se establecieron pautas que establecen la política nacional de prevención de tratamiento de desechos y la incorporación de la economía circular, con lo que el sector industrial y los distribuidores de productos ya se encuentra - desde un buen tiempo- en condiciones de comenzar a implementar estas directrices, no pudiendo aducir ignorancia. Por su propia cuenta, ya pueden comenzar a tomar las medidas necesarias de adaptación.

\section{Reacción comunal: ordenanza de la comuna de Providencia sobre plásticos de un solo uso}

A nivel de organismos públicos, de forma inédita, ciertos municipios están activando medidas en torno a los plásticos de un solo uso. ${ }^{33}$ La más explícita ha sido la Municipalidad de Providencia que el 29 de abril del año 2019 publicó la Ordenanza 182 de 2019 «sobre plásticos de un solo uso en Providencia». ${ }^{34}$ Entre otras, justifica la normativa en la Ley REP, en cuanto faculta a los municipios para diseñar e implementar medidas de prevención en la generación de residuos. A su vez tiene como orientación para el diseño de la regulación la guía elaborada por la ONU sobre Prohibición de plásticos de un solo uso. Conjunto de directrices para la sostenibilidad.

La regulación se enfoca en clases de productos, regulando bombillas y revolvedores, cubiertos plásticos, envases de comida de polipropileno, poliestireno y poliesti-

33. Emol, «Cuatro desconocidas 'comunas verdes' que destacan por sus iniciativas medioambientales», 6 de octubre de 2019, disponible en bit.ly/2Weg6K1.

34. No se puede dejar de mencionar que la comuna de Santiago también se encontraría en proceso de dictación de una ordenanza de esta naturaleza, la que estaría en condiciones de ser publicada prontamente, según informó el municipio, a raíz de consultas realizadas directamente a la Subdirección de Medio Ambiente. 
reno expandido, platos de poliestireno y poliestireno expandido para los que prohíbe los actos de comercio, distribución, expendio y/o entrega, bajo la una sanción entre 1 a 5 UTM.

Finalmente, la ordenanza contempla que el municipio celebrará con el comercio protocolos de gestión de residuos, para aquellos productos regulados que, por razones de seguridad de los consumidores o por la naturaleza del contenido, no puedan ser reemplazados por envases amigables con el medio ambiente y/o reutilizables.

\section{Inicitivas en discusión ante el Congreso}

El Congreso también se encuentra debatiendo en específico sobre los plásticos de un solo uso, sobre los que se podría hacer un rápido recorrido. La primera propuesta fue presentada por senadores de centroizquierda el 18 de mayo de 2015 (Boletín 10.054-12) que justificaba la medida considerando los océanos, la vida marina y los tóxicos que liberan estos materiales, a lo que agregó que en Chile «el 52\% del plástico es utilizado como un elemento comúnmente desechable y cuyo consumidor final y principal es un residente». ${ }^{35}$ No obstante, tiene una técnica poco feliz, pues termina refiriéndose solo a bolsas plásticas y films, con lo que además perdió su propósito con la dictación de la ley que regula la entrega de bolsas plásticas en Chile.

Ya con la Ley REP vigente, un segundo proyecto en discusión fue el Boletín 1130103, de 22 de junio de 2017, que propone modificar la Ley del Consumidor, para establecer la obligación de información a los consumidores, sobre el material de los envases plásticos. Se suma como tercer proyecto el Boletín 11578-12 de 17 de enero de 2018, que con un acabado desarrollo de la necesidad de la regulación destaca —entre otros- que el desecho de plástico hace del derecho a vivir en un medio libre de contaminación una pretensión «irrisoria», motivo por el cual - como técnica- establece una prohibición de «envases plásticos convencionales, de polietileno y polipropileno no biodegradables o no compostables», definiendo como «envase compostable» $\mathrm{o}$ «envase biodegradable», aquel que es biodegradable, generando dióxido de carbono, agua, y humus, a una velocidad similar a la de los materiales orgánicos, imponiendo la necesidad de ser certificados como tales.

Un cuarto proyecto en marcha fue el Boletín 11.651-12 de 29 de marzo de 2018, que entre sus fundamentos hace presente la necesidad de resguardar la salud y bienestar de las personas, para lo que establece un artículo único que solo se refiere a envases,

35. Indica adicionalmente el proyecto de ley: «la basura plástica — alrededor de 80 millones de toneladas- se encuentra flotando en los océanos Atlántico y Pacífico, destruyendo la vida marina, provocando reacciones tóxicas y liberando alteradores endocrinos - sustancias que son cancerígenas, mutagénicas o tóxicas para la reproducción-, nanopartículas y contaminantes orgánicos persistentes en los ecosistemas» (Congreso Nacional, Discusión legislativa sobre el Boletín 10.054-12, 2015). 
sancionando con multas que van desde las 10 a las 20 UTM. En una misma línea, se encuentra un quinto proyecto ingresado el 7 de junio de 2018 (Boletín 11.802-12) que también se enfoca únicamente en envases, prohibiendo su uso y comercialización, bajo las sanciones establecidas en la Ley REP.

A estos se sumó el Boletín 11.887-12 de 5 de julio del 2018, que añade un elemento internacional en su fundamentación. Explica que la política exterior del país se ha comprometido con una:

Participación activa en la agenda mundial para el desarrollo sustentable. Se ha puesto especial énfasis en incorporarse constructivamente a la red de acuerdos y tratados internacionales ambientales, los cuales constituyen una fuente importante del Derecho Ambiental Internacional y contribuyen a la generación de políticas de sustentabilidad de cada nación, estimulando además a la responsabilidad social de las empresas.

En esta línea y como un único artículo propone prohibir plásticos de un solo uso, sancionando con multas que van desde las 20 a las 50 UTM.

Un séptimo proyecto de ley (Boletín 12.633-12) ingresado por diputados de todo el espectro político el 15 de mayo de 2019, justificó la regulación considerando que «Chile produce más residuos sólidos municipales per cápita que México y Brasil, a pesar de que estos países lo superan ampliamente en población y en el tamaño de sus economías», lo que se conjuga con su difícil o imposible reciclado..$^{36}$ A esto agrega que alternativas «como los llamados oxobiodegradables, bioplásticos y compostables [...] no necesariamente pueden degradarse en ambientes naturales, si no que en condiciones específicas o industriales» lo que dificultan y aumentan el costo de su tratamiento, haciendo muy probable que terminen en «el mar al igual que los plásticos tradicionales» (Boletín 12.633-12).

Por ello, siguiendo la tendencia europea, propone regular una serie de productos: «vasos, tazas, tazones, cubiertos (tenedor, cuchara, cuchillo), mezcladores, bombillas, platos, envases o bandejas de comida preparada y sus envases accesorios, tapas, botellas iguales o menores de $500 \mathrm{cc}$ u otros que cumplan con el propósito de los productos individualizados» (Boletín 12.633-12), prohibiendo su entrega, cuando contengan poliestireno expandido o se trate de plásticos desechables que no se encuentren certificados. La certificación se referirá a su origen, degradabilidad en condiciones naturales y ausencia de toxicidad.

36. Establece el proyecto: «Hay productos que difícilmente serán reciclados porque son pequeños o livianos, o de limitado volumen, y porque tienen bajo valor económico en comparación con sus elevados costos de recolección, clasificación y reciclaje. Por este motivo, estudios internacionales estiman que cerca de un 30\% de los plásticos debe ser reemplazado por otros materiales ya que no serán reciclados. En definitiva, estos objetos pequeños o livianos, y en especial aquellos de plástico, solo podrán ingresar a la economía circular mediante la prevención de su generación y el fomento de su reutilización» (Boletín 12.633-12). 
Un octavo Boletín 12639-12 de 15 de mayo de 2019 que, citando la normativa europea reciente, hace hincapié en el movimiento regulatorio actual: «[en] el escenario internacional y nacional actual, la tendencia legislativa debe promover la prohibición de las prácticas económicas no sostenibles y que generan impactos considerables al Medio Ambiente, prefiriendo la proliferación de actividades y prácticas sostenibles y ecológicas» (Boletín 12.633-12). Por este motivo, este proyecto propone prohibir: «el uso, entrega y comercialización de envases, botellas plásticas y bolsas plásticas no reciclables, utensilios plásticos y productos de un solo uso o desechables» (Boletín 12.633-12). Para esto, entrega la fiscalización a los municipios, con una sanción de hasta 10 UTM por cada producto entregado.

Finalmente, un noveno y último proyecto en tramitación es el Boletín 12641-12, que refundiendo otras iniciativas, ${ }^{37}$ destaca la crisis del plástico, la afectación a recursos de agua dulce y alimentos provenientes del mar, por lo que propone una prohibición que regirá 5 años después de publicada la Ley:

Se prohíbe la venta o entrega gratuita de platos, recipientes, vasos, cubiertos, bombillas y cualquier otro artefacto cuyo material de elaboración sea el plástico u otro material de difícil biodegradación y su vida útil termine con un solo uso o consumo o con un número reducido de usos o consumos (Boletín 12641-12).

Este proyecto, hasta el momento en su discusión ante el Senado, giró y focalizó su atención al uso de plásticos en establecimientos de alimentos y las botellas plásticas. Así, estaría indicando que i) para el consumo dentro de estos establecimientos, no se podrá utilizar «vasos, tazas, tazones, cubiertos (tenedor, cuchara y cuchillo), palillos, pocillos, mezcladores, bombillas, platos, cajas, copas, envases de comida preparada, bandejas, sachets, individuales y tapas que no sean de botellas, en tanto no sean reutilizables» (Boletín 12641-12). Si el consumo se realiza fuera del local ii):

[Se] prohíbe la entrega, a cualquier título, por parte de los establecimientos, de productos de un solo uso, salvo que el consumidor expresamente los solicite. Los establecimientos que entreguen productos de un solo uso deberán informar a los consumidores sobre la manera adecuada de valorizar los residuos en los que se transformarán dichos productos y sensibilizar a los consumidores sobre la importancia de dicha valorización (Boletín 12641-12).

Finalmente, en cuanto a las botellas plásticas, este proyecto en lugar de imponer un sistema de botellas retornables que reduzcan el volumen de estos desechos, opta por permitir su comercialización cuando provengan de plásticos reciclados en Chile:

37. Durante su tramitación, se refundió a este proyecto, los boletines 11429-12, 12561-12, 11809-12 y 12633-12. 
Las botellas plásticas desechables que se comercialicen por cualquier persona natural o jurídica, sean o no establecimientos de expendio de alimentos, deberán estar compuestas por un porcentaje de plástico que haya sido recolectado y reciclado dentro del país, en las proporciones que determine el reglamento de esta ley (Boletín 12641-12).

\section{A modo de conclusión: Algunas observaciones que promueven la prohibición de los plásticos de un solo uso: urgencia, falta de novedad, progresión, horizontalidad y respeto de derechos fundamentales}

Los antecedentes revisados muestran que los desechos plásticos están profundizando la crisis climática y generando serias vulneraciones a derechos fundamentales, todo ello con un fuerte impacto económico. Al mismo tiempo, principios constitucionales y ambientales moldean la respuesta ante el problema, en un análisis que no solo le cabe realizar al legislador al momento de profundizar la normativa chilena, sino que a todos los actores, públicos o privados. En particular y sobre la caracterización realizada a lo largo de este trabajo, me gustaría compartir siete reflexiones:

\section{Statu quo y urgencia de medidas ambiciosas}

El statu quo ya se conoce. De nada hacer y de mantenerse la situación sin medidas, los datos muestran que el consumo de plástico y su uso indiscriminado no ha disminuido en el último período, sino que por el contrario ha y seguirá en aumento. Aun cuando sería deseable un cambio en el comportamiento de todos los actores, parece que no existe una reacción fuerte de autoregulación, pese a que los efectos de los desechos del plástico se conocen desde un buen tiempo.

Adicionalmente, parece que la cuestión no solo pasa por una decisión de los consumidores que en muchas ocasiones reciben productos con una cantidad innecesaria de este material, no teniendo mayores alternativas, lo que pone la atención en los comercializadores y productores que hasta ahora no muestran una mayor reacción.

No perder de vista los sujetos y bienes jurídicos involucrados en la problemática del plástico

La regulación de los desechos plásticos y la reducción de plásticos de un solo uso descansa en un componente constitucional muy fuerte. La discusión no solo es vida y salud versus el uso de un material, sino que - como se ha visto- la protección de la economía y otras actividades, el uso de recursos públicos que se malgastan retirando desechos, los efectos de la contaminación sobre el medio ambiente y la protección de la naturaleza y las generaciones futuras son también parte de los elementos que justifican la intervención estatal. 
El movimiento internacional y comparado ha recalcado la necesidad de un nuevo paradigma justificado en una economía circular, que resalta la imposibilidad de sostener en el tiempo un modelo de producción-consumo-desecho, siendo imprescindible que los desechos plásticos se eviten, y respecto de aquellos que no pueden ser evitados, se mantengan en un sistema de valorización y comercialización, lo que a su vez promueve nuevos rubros y empleos.

Así, ante los clásicos temores sobre los efectos económicos negativos que puede significar prohibir un producto o limitarlo enormemente, aparece este llamado a la realidad, que transparenta que, en el estado actual, se están perdiendo muchos recursos y afectando derechos fundamentales con productos que se usan una vez y luego se tiran.

\section{La prevención de desechos antes que el reciclaje: una eventual regresión}

Por lo caro y difícil de su manejo, la tendencia comparada e internacional destaca la necesidad de prevenir la generación de desechos. Pero, esta consideración también se encuentra en el derecho interno: tratados suscritos por Chile, en los que ya se afirma esta visión, reiterada en la Ley REP se vincula indisolublemente con una lógica de un desarrollo sustentable.

Considerando la discusión del Congreso, la solución no va solo por sustituir plásticos por otros certificados que tengan ciertas características, porque termina siendo una solución difícil de implementar y muy costosa. La separación de cada uno de ellos, lo dificultosa de su biodegradabilidad, la necesidad de una industria altamente desarrollada capaz de procesar todos los tipos de plástico, hacen difícil e ilusoria una posibilidad realista de reciclaje. Además, esta solución parece contraria a los compromisos suscritos relacionados con la disminución de gases con efecto invernadero con ocasión de los desechos, la prevención de desechos y el desarrollo sustentable.

El caso europeo y el caso chino destacan precisamente la trampa de intentar solucionar esta problemática con solo reciclaje, porque la experiencia ha mostrado que la recolección y separación es difícil y que el monto de plásticos que se puede terminar aprovechando es muy menor incluso al recolectado. Con ello, se está perdiendo como sociedad terrenos que terminan siendo destinados a basurales, se está pagando grandes cantidades por la mantención de ese sistema, en costos que generalmente asumen los municipios, y no se logra detener la llegada de estos desechos al ambiente, ríos, playas y océanos, lugar en los que por su diseño, causan estragos en la naturaleza.

Parece una medida regresiva renunciar al principio de prevención en la generación de desechos e intentar poner todas las esperanzas únicamente en el sistema de reciclaje. 


\section{Transversalidad de las restricciones a los plásticos}

Aun tomando distintos puntos de vista, solo es posible concluir que la restricción de los plásticos de un solo uso es un fenómeno transversal. Los efectos no se restringen a un territorio, sino que la crisis del plástico tiene una escala mundial. La regulación va desde la ONU hasta pequeñas comunas o provincias. Desde oriente (China e India) hasta occidente. Es más, en Chile, las iniciativas se han presentado desde partidos de todo el espectro político.

Si se mira al desecho plástico, también se puede hablar de transversalidad. Con independencia de su origen (biodegradable o no), uso, duración u otro, está siendo de todos modos regulado, sea para prohibirlo en algunos casos, etiquetarlo y diferenciarlo en otros, o para prevenirlo en los casos posibles.

Esta transversalidad también genera paradojas. Si una empresa trasnacional está fuertemente regulada en un país extranjero, y utiliza productos reutilizables o biodegradables allá, bueno, ¿por qué no podría hacerlo acá, si ya es parte de su negocio?

Así, considerando este aparente consenso nacional e internacional, sería ideal que jornadas internacionales como la COP permitieran consolidar avances y actualizar compromisos a escala global, pues incluso las medidas nacionales parecen no ser suficientes. Por lo demás, un sistema mundial de reciclaje, que opere con certificados similares, etiquetados y estándares de producto, pueden impulsar una economía circular mundial, tal como ha vislumbrado en su zona la Unión Europea.

\section{Las limitaciones no son un fenómeno novedoso}

Pese a lo urgente de lograr una reducción de los desechos plásticos, también se puede rescatar lo poco novedoso de la propuesta. Ni en el derecho chileno (ya el Código Civil reconoce la posibilidad de prohibir por objeto o causa ilícita una serie de contratos), ni en materia internacional se está ante un fenómeno sin precedentes. El plástico desde bastante tiempo ha tenido una regulación, sea por su contacto con alimentos, sea por su toxicidad.

Adicionalmente, los compromisos internacionales suscritos por Chile, la lectura constitucional, los principios contenidos en la Ley REP y la incipiente actuación municipal apuntan a la medida, de forma horizontal (productores, consumidores y distribuidores) y vertical (estatal). La industria ha tenido ocasión de advertir la vigencia del principio de prevención de desechos y el desarrollo sustentable, por lo que considerando todos estos antecedentes sería difícil que reclamara que se está ante una regulación inesperada, para la que no se encuentra preparada. El solo hecho de advertir los movimientos regulatorios internacionales, y los avances legislativos los dejan en posición de aproximarse y comenzar la adaptación a nuevos escenarios, en los que sería ideal una pronta actuación de oficio. 


\section{La existencia de una guía elaborada por la ONU} simplifica enormente la adopción de una política

Sin perjuicio que las grandes directrices sobre el manejo de residuos ya fueron rescatadas en la Ley REP, la publicación de una guía por parte de la ONU simplifica la implementación de medidas concretas y permite advertir las correcciones necesarias que pueden ser implementadas.

Así, el énfasis en la pluralidad de medidas, la necesidad de involucrar a todos los actores y la revisión constante de los resultados, son elementos claves que se deben chequear para ver en qué medida se implementa para el control de desechos plásticos, sin un mayor costo. Ya se sabe en buena medida cuáles son los caminos que se pueden seguir.

Siguiendo esta experiencia procesada por ONU, es bueno advertir que uno de los mecanismos es iniciar el proceso con ciertos tipos de plástico, típicamente envases, botellas, bombillas, cotonitos, entre otros. Por ello, cuando se contrastan los productos que se pretenden regular por la discusión legislativa actual, da la impresión que la medida es un poco restrictiva al considerar solo ciertos plásticos entregados en restaurantes y no aprovechar la oportunidad para regular algunos otros desechos o envases.

\section{Todos estamos en condiciones de implementar medidas}

A diferencia de otras materias, en que la discusión se encuentra concentrada ante un órgano, en esta materia la potestad para regular y avanzar en la materia está en los municipios, el Ministerio del Medio Ambiente, el Congreso, y los órganos de la administración del Estado que para sus respectivos servicios pueden adoptar medidas como la reducción de botellas plásticas. Todos con un grado mayor o menor influencia territorial, pueden limitar su uso de plásticos innecesarios y/o adoptar medidas.

Pero esta reacción también es posible desde consumidores (con mayor dificultad, por lo complejo que es obtener productos en esta lógica) y los productores y comercializadores. No se puede dejar de observar que, aun cuando falte la dictación de los reglamentos respectivos, al menos desde el año 2016 con la publicación de la Ley REP, se establecieron pautas que establecen la política nacional de prevención de tratamiento de desechos y la incorporación de la economía circular, con lo que el sector industrial ya se encuentra - desde un buen tiempo- en condiciones de comenzar a implementar estas directrices, no pudiendo aducir ignorancia. Por su propia cuenta, ya pueden adoptar medidas necesarias, más aún si consideran los bienes jurídicos involucrados, como ha sido insistentemente señalado. 


\section{Referencias}

Biblioteca del Congreso Nacional (2015). Discusión legislativa sobre el Boletín 10.054-12 de 18 de mayo de 2015. Disponible en bit.ly/3rouEFg.

Biblioteca del Congreso Nacional (2018). Boletín 11.887-12 de 5 de julio del 2018. Disponible en bit.ly/3rokiVR.

Biblioteca del Congreso Nacional (2019). Boletín 12.639-12 de 15 de mayo del 2019. Disponible en bit.ly/34Eq7ov.

Biblioteca del Congreso Nacional. Historia de la Ley 20.920. Disponible en BIT.LY/3GKPLLJ.

Dalberg Global Development Advisors y The University Of Newcastle (2019). Naturaleza sin plástico. Evaluación de la ingestión humana de plásticos presentes en la naturaleza. Disponible en bit.ly/37ZLMs\%.

Gennip, Simon Jan Van, Boris Dewitte, Véronique Garçon y Martin Thiel (2019). «In search for the sources of plastic marine litter that contaminates the Easter Island Ecoregion» Scientific reports 9 (19662). DOI: 10.1038/s41598-019-56012-x.

Mercosur (1992). Resolución 3/92, de 30 de marzo de 1992, que establece Criterios generales de envases y equipamientos alimentarios en contacto con alimentos. Disponible en bit.ly/3mPacK6.

Ministerio del Medio y Ambiente y Espacio Público Argentino (2019). Resolución 816 del Ministerio del Medio Ambiente y Espacio Público, de 22 de mayo de 2019. Disponible en bit.ly/3mELOLe.

Ministerio Del Medio Ambiente y COP 25 (2019). Océano y Cambio Climático. 50 preguntas y respuestas. Disponible en bit.ly/384Hxva.

Municipalidad de Providencia (2019). Ordenanza 182/2019, sobre plásticos de un solo uso en Providencia. Disponible en bit.ly/2IR $31 \mathrm{C} 8$.

OCeAna (2020). Propuesta para limitar la generación de productos desechables y regular los plásticos. Disponible en bit.ly/3475Do8.

ONU Medio Ambiente (2018). Plásticos de un solo uso: Una hoja de ruta para la sostenibilidad. Disponible en bit.ly/34DjFOm.

Perú, Ministerio del Medio Ambiente (2018). Ley 30.884. que regula el plástico de un solo uso y los recipientes o envases descartables». Disponible en bit.ly/2KgGfFf.

Téllez, Alejandra (2012). «La complejidad de la problemática ambiental de los residuos plásticos: una aproximación al análisis narrativo de política pública en Bogotá». Tesis de Maestría, Universidad Nacional de Colombia. Disponible en bit. ly/3mMidtg.

Unión Europea (1994). Directiva 94/62/CE del Parlamento Europeo y del Consejo de 20 de diciembre de 1994. Disponible en bit.ly/37InILa.

Unión Europea (2008). Directiva 2008/98/CE del Parlamento Europeo y del Consejo, de 19 de noviembre de 2008. Disponible en bit.ly/38CwUjM. 
Unión Europea (2018). Directiva (UE) 2018/851 del Parlamento Europeo y del Consejo de 30 de mayo de 2018. Disponible en bit.ly/3hfveQV.

Unión Europea (2018). Directiva (UE) 2018/852 del Parlamento Europeo y del Consejo de 30 de mayo de 2018. Disponible en bit.ly/zayoexk.

Unión Europea (2019). Directiva (UE) 2019/904 del Parlamento Europeo y del Consejo 5 de junio de 2019.Disponible en bit.ly/3nGsnTz.

United Nation Environment Program (2018) Legal Limits on Single-Use Plastics and Microplastics: A global Review of National Laws and Regulations. Disponible en bit.ly/2Kgeuwx.

United Nation Environment Assembly (2019). Draft Ministerial Declaration of the 2019 United Nations Environment Assembly "Innovative solutions for environmental challenges and sustainable consumption and production». Disponible en bit. ly/3mfdypz.

\section{Sobre el autor}

Camilo Andrés Cornejo Martínez es abogado de la Universidad de Chile, Magister en Derecho (c) mención en Derecho Público de la misma Universidad. Profesor Instructor del Departamento de Enseñanza Clínica de la Facultad de Derecho de la Universidad de Chile. Su correo electrónico es camilo.cornejo@ug.uchile.com.

https://orcid.org/0000-0001-9433-0817. 
La Revista de Derecho Ambiental, del Centro de Derecho Ambiental de la Facultad de Derecho de la Universidad de Chile, es un espacio de exposición y análisis en el plano académico del derecho ambiental. Su contenido se presenta a través de doctrina, jurisprudencia y recensiones, y aborda diversas materias relacionadas con la gestión, institucionalidad y herramientas de protección ambiental y desarrollo sustentable. Se presentan artículos de diferentes autores y autoras en los que se analizan y abordan casos y temas jurídico-ambientales de creciente interés y actualidad.

\author{
DIRECTORA \\ Valentina Durán Medina \\ EDITORES \\ Jorge Ossandón Rosales \\ y Antonio Pulgar Martínez \\ SITIO WEB \\ revistaderechoambiental.uchile.cl \\ CORREO ELECTRÓNICO \\ revistada@derecho.uchile.cl \\ LICENCIA DE ESTE ARTÍ́CULO \\ Creative Commons Atribución Compartir Igual 4.o Internacional
}

La edición de textos, el diseño editorial

y la conversión a formatos electrónicos de este artículo

estuvieron a cargo de Tipográfica

(www.tipografica.io) 